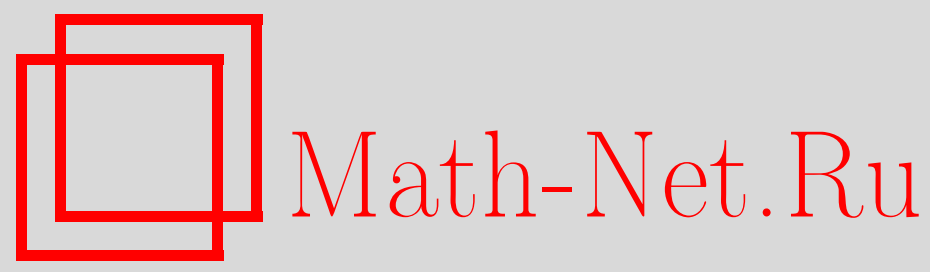

Д. И. Савельев, Об изоморфизме отношений, вкладывающихся друг в друга, Матем. заметки, 2003, том 74, выпуск 4, 573-589

DOI: https://doi.org/10.4213/mzm294

Использование Общероссийского математического портала Math-Net.Ru подразумевает, что вы прочитали и согласны с пользовательским соглашением http://www.mathnet.ru/rus/agreement

Параметры загрузки:

IP: 35.173 .219 .12

26 апреля 2023 г., 10:30:38

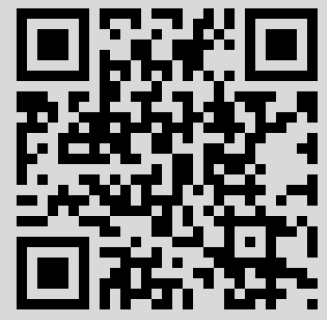




\section{ОБ ИЗОМОРФИЗМЕ ОТНОШЕНИЙ, ВКЛАДЫВАЮЩИХСЯ ДРУГ В ДРУГА}

\section{Д. И. Савельев}

В статье рассматриваются аналоги теоремы Кантора-Бернштейна для множеств с бинарными отношениями. В п. 1 доказан некоторый аналог этой теоремы, верный для произвольных бинарных отношений; в п. 2 дано одно его приложение; в п. 3 рассмотрен класс отношений, для которых верен непосредственный аналог теоремы Кантора-Бернштейна и близкий к нему класс точных отношений, доказана их замкнутость относительно некоторых операций.

Библиография: 17 названий.

Введение. В этом пункте мы напомним основные понятия, касающиеся релящионных систем, и сформулируем основные задачи данной работы.

Пусть $A$ - множество (или класс), $\mathscr{R} \subseteq A \times A$ - бинарное отношение на $A$. Упорядоченная пара $(A, \mathscr{R})$ называется реляционной системой (см. $[1$, с. 91], $[2$, с. 81]) или моделью, в которой вьполнены аксиомы, характеризующие свойства $\mathscr{R}$. Будем, как обычно, писать $x \mathscr{R} y$ вместо $(x, y) \in \mathscr{R}$.

Отношение $\mathscr{R}^{-1}$, обратное к отношению $\mathscr{R}$, есть $\{(y, x): x \mathscr{R} y\} ;$ суперпозиция $\mathscr{S}$ о $\mathscr{R}$ отношений $\mathscr{R}$ и $\mathscr{S}$ есть $\{(x, z): \exists y(x \mathscr{R} y \wedge y \mathscr{S} z)\}$. Отношение $\mathscr{R}=0$ - nyстое, a $\mathscr{R}=A \times A-$ универсальное; $\mathscr{R}=\Delta_{A}$ (диагональ в $\left.A \times A\right)$-отношение равенства; мы будем писать просто $\Delta$, опуская индекс $A$. Отношение $\mathscr{R}$ рефлексивно, если $\Delta \subseteq \mathscr{R} ; а н$ тирефлексивно, если $\mathscr{R} \cap \Delta=0 ;$ симметрично, если $\mathscr{R}=\mathscr{R}^{-1} ;$ антисимметрично, если $\mathscr{R} \cap \mathscr{R}^{-1}=\Delta ;$ асимметрично, если $\mathscr{R} \cap \mathscr{R}^{-1}=0 ;$ транзитивно, если $\mathscr{R} \circ \mathscr{R} \subseteq \mathscr{R} ;$ связно, если $\mathscr{R} \cup \mathscr{R}^{-1}=A \times A$. (Легко проверить, что отношение $\mathscr{R}$ асимметрично, если и только если дополнительное к нему отношение $(A \times A) \backslash \mathscr{R}$ связно.) Каждьй граф можно отождествить с антирефлексивным симметричным отношением. Рефлексивное и транзитивное отношение назьвается предпорядком; добавляя аксиому симметрии или антисимметрии, получим соответственно отношение әквивалентности или отношение порядка. Строгим порядком называется транзитивное асимметричное отношение. Если порядок (или строгий порядок) связньй, он называется линейным. Реляционные системы с отношением (строгого, линейного, пред-) порядка обычно назьвают ( $c m p o-$ го, линейно, пред-) упорядоченными множествами. Отношение ЯR фундированно, если любое его подмножество $A_{0} \subseteq A$ содержит $\mathscr{R}$-минимальный элемент $x_{0}$, т.е. такой $x_{0} \in A_{0}$, что $\neg \exists x \in A_{0}\left(x \mathscr{R} x_{0}\right)$; в частности, отсюда следует, что не существует бесконечных "убьваюших" последовательностей вида $\ldots \mathscr{R} x_{n} \mathscr{R} \ldots \mathscr{R} x_{1} \mathscr{R} x_{0}$. Фундированные линейно упорядоченные множества назьвают вполне упорядоченными. Важным примером другого рода является $\in$ - отношение принадлежности. Считается, что его свойства описываются аксиомами теории множеств. 
Сужение отношения $\mathscr{R}$ на подмножество $A_{0} \subseteq A$ есть отношение $\mathscr{R}_{0}=\mathscr{R} \cap\left(A_{0} \times A_{0}\right)$; сужение реляционной системы $(A, \mathscr{R})$ на это подмножество есть реляционная система $\left(A_{0}, \mathscr{R}_{0}\right)$, которая называется ее подсистемой. Подсистема называется выпуклой или отрезком, если $\forall x_{0}, x_{1} \in A_{0} \forall x \in A\left(x_{0} \mathscr{R} x \wedge x \mathscr{R} x_{1} \Rightarrow x \in A_{0}\right)$. Отрезок будет финальным, если $\forall x_{0} \in A_{0} \forall x \in A\left(x_{0} \mathscr{R} x \Rightarrow x \in A_{0}\right)$; аналогично определяется инициальньй отрезок. Скажем, что отрезок нетривиальны й, если сужение отношения $\mathscr{R} \backslash \Delta$ на него непусто.

НЕКОТОРЫЕ СВоЙСТВА И ПРИМЕРЫ. В системе с пустьп отношением (или с отношением равенства) все подсистемы являются тривиальными отрезками. В системе с универсальным отношением есть только один (несобственный) отрезок, содержащий более одной точки. В системе со связным отношением отрезок будет нетривиальньм, если и только если он содержит более одной точки. Отношение антисимметрично, если и только если любая точка образует отрезок; транзитивно, если и только если любой отрезок есть пересечение (наименьших содержащих его) инициального и финального.

Система $(A, \mathscr{R})$ плотная (как и отношение $\mathscr{R})$, если "между" любьми различными ее точками, связанными отношением $\mathscr{R}$, найдется другая точка; т.е. если положить $\mathscr{S}=\mathscr{R} \backslash \Delta$, плотность отношения $\mathscr{R}$ означает, что $\mathscr{S} \subseteq(\mathscr{S} \circ \mathscr{S}) \cup(\mathscr{S} \circ \mathscr{S})^{-1}$. Заметим, что $\mathscr{R}$ плотно и транзитивно, если и только если $\mathscr{S}=\mathscr{S}$ о $\mathscr{S}$. Система разрежсена, если она не содержит плотных подсистем.

Инъекция $f: A \rightarrow B$ назьвается вложсением системы $(A, \mathscr{R})$ в систему $(B, \mathscr{S})$, если $\forall x, y \in A(x \mathscr{R} y \Leftrightarrow f(x) \mathscr{S} f(y))$. Вложение, являющееся биекцией, назьвается изоморфизмом; изоморфизм системы на себя назьвается автоморфизмом; системы, между которыми существует изоморфизм назьваются изоморфными. Ясно, что изоморфизм устанавливает между релящионными системами отношение эквивалентности, а вложение предупорядочивает их реляиионные типы - классы эквивалентности по изоморфизму. Мы будем обозначать релящионные типы греческими буквами $\alpha, \beta, \rho, \sigma, \ldots$. Мощности можно отождествить с типами систем, в которых отношение пусто или универсально. Типы линейно упорядоченных множеств назьваются порядковыми типамu; типы вполне упорядоченных множеств - ординалами. С другой стороны, мы будем, когда это будет удобно, отождествлять ординалы с множествами, вполне упорядоченными отношением $\in$, а мощности - с кардиналами, т.е. начальными ординалами (следуя хорошо известной идее фон Неймана; см., например, [16]).

Примеры. На множестве $2=\{0,1\}$ существуют ровно три типа систем с антирефлексивным отношением: тип системы с пустым отношением, с отношением $\{(0,1)$, $(1,0)\}$ и с отношением $\{(0,1)\}$ строгого линейного порядка (последняя система представляет собой ординал 2 с сужением отношения $\in$ на него, и изоморфна оставшейся системе с отношением $\{(1,0)\})$. На множестве $3=\{0,1,2\}$ существуют ровно два типа систем с асимметричным связньм отношением: тип системы со строгим линейным порядком $\{(0,1),(1,2),(0,2)\}$ и с отношением $\{(0,1),(1,2),(2,0)\}$, которое будем назьвать ииклическим. Очевидно, первая система разрежена и имеет два собственных нетривиальных отрезка, а вторая плотная и не имеет таких отрезков.

Пусть множества $A_{b}$ при различных $b \in B$ не пересекаются. Обозначим через $\bigsqcup$ и $\sqcup$ (обобщенное и бинарное соответственно) дизъюнктное объединение. Система

$$
(C, \mathscr{T})=\sum_{(B, \mathscr{S})}\left(A_{b}, \mathscr{R}_{b}\right)
$$


есть (обобщенная) сумма реляиионных систем $\left(A_{b}, \mathscr{R}_{b}\right)$ по реляционной системе $(B, \mathscr{S})($ см. [2, с. 60, 81]), если

$$
C=\bigsqcup_{b \in B} A_{b} \quad \text { и } \quad \mathscr{T}=\bigsqcup_{b \in B} \mathscr{R}_{b} \sqcup \bigsqcup_{b^{\prime}(\mathscr{S} \backslash \Delta) b^{\prime \prime}}\left(A_{b^{\prime}} \times A_{b^{\prime \prime}}\right) .
$$

Заметим, что сумма не изменится, если вместо $\mathscr{S}$ взять антирефлексивное отношение $\mathscr{S} \backslash \Delta$ (или рефлексивное $\mathscr{S} \cup \Delta$ ). Легко проверить, что такое обобщенное сложение "обобщенно ассоциативно" в следующем смысле:

$$
\sum_{(C, \mathscr{T})} \sum_{\left(B_{c}, \mathscr{S}_{c}\right)}\left(A_{b_{c}}, \mathscr{R}_{b_{c}}\right)=\sum_{\sum_{(C, \mathscr{T})}\left(B_{c}, \mathscr{S}_{c}\right)}\left(A_{b_{c}}, \mathscr{R}_{b_{c}}\right)
$$

Многие свойства (как симметричность, асимметричность, транзитивность, связность, плотность и т.п.) сохраняются при переходе к сумме, если ими обладали все слагаемые и система, по которой они суммировались. В п. 3 мы будем обсуждать сохранение некоторых интересующих нас свойств.

Скажем, что операции сложения одного типа, если системы, по которым суммируются слагаемые, имеют один тип. Из сказанного вьше следует, что существуют ровно три типа бинарного сложения: по системе с пустым отношением, с универсальным отношением и с отношением линейного порядка; они назьваются соответственно кардинальным, квадратным и ординальным сложениями (см. [2, с. 1-2]). Заметим, что квадратное и кардинальное сложения коммутативны, а ординальное некоммутативно. Бинарное сложение одного фиксированного типа ассоциативно:

$$
((A, \mathscr{R})+(B, \mathscr{S}))+(C, \mathscr{T})=(A, \mathscr{R})+((B, \mathscr{S})+(C, \mathscr{T}))
$$

где + означает любое сложение одного и того же типа; однако если + и + ${ }^{\prime}-$ какие-нибудь два бинарных сложения различных типов и 1 обозначает фиксированную одноточечную систему, то очевидно, что $(1+1)+^{\prime} 1 \neq 1+\left(1+^{\prime} 1\right)$. Эти бинарные операции формализуются в кардинальных и ординальных алгебрах, развитых Тарским соответственно в [3] и [2].

Система $(C, \mathscr{T})=(A, \mathscr{R}) \cdot(B, \mathscr{S})$ есть (бинарное) ординальное произведение систем $(A, \mathscr{R})$ и $(B, \mathscr{S})$, если $C=A \times B$, а $\mathscr{T}$ получается из $\mathscr{R}$ и $\mathscr{S}$ антилексикографически (по последнему различию), т.е.

$$
\forall\left(x_{0}, y_{0}\right),\left(x_{1}, y_{1}\right) \in A \times B \quad\left(\left(x_{0}, y_{0}\right) \mathscr{T}\left(x_{1}, y_{1}\right) \Leftrightarrow y_{0}(\mathscr{S} \backslash \Delta) y_{1} \vee\left(y_{0}=y_{1} \wedge x_{0} \mathscr{R} x_{1}\right)\right) .
$$

Заметим, что та же система получится, если взять лексикографическое произведение и $\mathscr{S}^{-1}$ вместо $\mathscr{S}$. Легко видеть, что ординальное произведение $(A, \mathscr{R}) \cdot(B, \mathscr{S})$ равно сумме $\sum_{(B, \mathscr{S})}\left(A_{b}, \mathscr{R}_{b}\right)$, где $A_{b}=A \times\{b\}$ для всех $b \in B$ и $\mathscr{R}_{b}$ определены естественным образом. (Заметим, что даже если все слагаемые в сумме $\sum_{(B, \mathscr{S})}\left(A_{b}, \mathscr{R}_{b}\right)$ изоморфны $(A, \mathscr{R})$, мы не можем получить изоморфизм между этой суммой и произведением $(A, \mathscr{R}) \cdot(B, \mathscr{S})$ без $\mathrm{AC}$ (аксиомы выбора).) Системы $((A, \mathscr{R}) \cdot(B, \mathscr{S})) \cdot(C, \mathscr{T})$ и $(A, \mathscr{R}) \cdot((B, \mathscr{S}) \cdot(C, \mathscr{T}))$ не совпадают (их основные множества $(A \times B) \times C$ и $A \times(B \times C)$ различны), но очевидным образом изоморфны.

Все эти операции переносятся на релящионные типы, так как тип образующейся системы не меняется при замене исходных систем на изоморфные им. В силу сказанного выше ординальное умножение типов ассоциативно. 
ЗАмЕчАниЕ. Аналогично можно определить общее антилексикографическое произведение $\prod_{(B, \mathscr{S})}\left(A_{b}, \mathscr{R}_{b}\right)$, частным случаем которого является степень $(A, \mathscr{R})^{(B, \mathscr{S})}$. Легко видеть, что для связности произведения не достаточно связности $\mathscr{S}$ и всех множителей: нужно, чтобы точки произведения всегда имели "последнее различие", т.е. чтобы $B$ было вполне упорядоченно отношением $\mathscr{S}^{-1}$. Другой распространенной конструкцией является кардинальное произведение, отношение в котором определяется всеми $\mathscr{R}_{b}$ и (только) мощностью $B$. Очевидно, оно будет связным, только если все $\mathscr{R}_{b}$, кроме разве что одного, универсальны. Можно предложить следующую общую конструкцию (включающую, в частности, оба этих случая). Пусть $C=\prod_{b \in B} A_{b}-$ декартово произведение, и $f: C \times C \rightarrow \mathscr{P}(B)$. Система $(C, \mathscr{T})=\prod_{(B, \mathscr{S}), f}\left(A_{b}, \mathscr{R}_{b}\right)$ есть (обобщенное) произведение систем $\left(A_{b}, \mathscr{R}_{b}\right)$ по системе $(B, \mathscr{S})$ и отобрахсению $f$, если

$$
\forall x^{\prime}, x^{\prime \prime} \in C \quad\left(x^{\prime} \mathscr{T} x^{\prime \prime} \Leftrightarrow \forall b \in f\left(x^{\prime}, x^{\prime \prime}\right) \quad\left(x^{\prime}(b) \mathscr{R}_{b} x^{\prime \prime}(b)\right)\right) .
$$

В этой работе мы не будем использовать такие произведения.

Рассмотрим ситуацию, когда две реляционные системы вкладываются друг в друга:

УСЛовиЕ $(*)$. Система $(A, \mathscr{R})$ изоморфна сужению системы $(B, \mathscr{S})$ на некоторое $B_{0} \subseteq B$, а система $(B, \mathscr{S})$ - сужению системы $(A, \mathscr{R})$ на некоторое $A_{0} \subseteq A$.

Классическая теорема Кантора-Бернштейна, впервые (1887 г.) доказанная Дедекиндом (см. [4, т. 3, с. 447]), утверждает, что если изоморфизмы суть просто биекции, то условие $(*)$ влечет изоморфизм систем $(A, \mathscr{R})$ и $(B, \mathscr{S})$ (см., например, $[16$, с. 85]).

Классическая теорема Кантора-Бернштейна говорит, что если изоморфизмь суть просто биекции, то условие (*) влечет изоморфизм систем $(A, \mathscr{R})$ и $(B, \mathscr{S})$ (см., например, [16, с. 85]; впервые (1887) она была доказана Дедекиндом, см. [4, т. 3, с. 447]). Иначе говоря, на мощностях предпорядок по вложению является порядком. Конечно, в общем случае аналогичное заключение неверно; тривиальньм контрпримером служат открытый и замкнутьй отрезки действительной прямой с обычным отношением линейного порядка. Если мы все же хотим иметь какой-то аналог этой теоремы для более общих видов релящионных систем, у нас есть две возможности. Можно, во-первых, рассмотреть те системы, для которых это заключение остается верным. Это мы сделаем в п. 3. С другой стороны, можно попытаться усилить условие $(*)$, чтобы заключение стало верным для всех систем. Эта возможность рассмотрена в следующем пункте.

ЗАмЕчАния. (1) Банах доказал следующую теорему (см. [1, с. 194]). Ecли дань инвекиии $f: A \rightarrow B$ и $g: B \rightarrow A$, то каждое из множсеств $A$ и $B$ можсно разбить на два множества так, что сужения отображений $f$ и $g$ на них будут биекииями. Тогда отображение $h$, являющееся объединением сужения $f$ и отображения, обратного к сужению $g$, будет биекцией из $A$ на $B$, что дает теорему Кантора-Бернштейна. Очевидно, если $f$ и $g$-вложения, то соответствующие сужения (но, вообще говоря, не $h$ ) будут изоморфизмами.

(2) Хорошо известное альтернативное доказательство теоремы Кантора-Бернштейна использует метод неподвижной точки, примененньй к полной решетке вех подмножеств данного множества (см. [1; ib.]; это доказательство, как и теорема, обсуждаемая в п. 1 , принадлежит Линденбауму и Тарскому [17]). Преимущество такого доказательства в том, что оно не использует AInf (аксиому бесконечности). Хотя теорема о неподвижной точке верна для любой полной решетки (Тарский), мы не можем применить этот метод 
к релящионным системам; в самом деле, они (как и их типы) не образуют даже решетки по вложению (например, двухэлементное множество $\left\{\omega, \omega^{*}\right\}$ не имеет наибольшей нижней грани).

Разумеется, все рассуждения этой статьи могут быть формализованы в ZF (теории множеств Цермело-Френкеля). Более того, AInf использована только в пп. 1 и 2; результаты п. 3 не зависят от нее (исключая второе утверждение леммы 6(2) и некоторые примеры в тексте). Мы нигде не используем АC, кроме п. 2, где доказательство теоремы 2 зависит от счетного выбора и второе утверждение ее следствия зависит от несчетного выбора (ср. замечание в конце п. 2).

1. Теорема Линденбаума-Тарского. Линденбаум нашел следующее замечательное

УТВЕРЖДЕНИЕ (см. [17] или [5, с. 22], [6, с. 248]). Если одно линейно упорядоченное множество изоморфно финальному отрезку другого, а другое - инициальному отрезку первого, то оба мнохсества изоморфны.

Рассматривая доказательство этой теоремы, можно заметить, что фактически используется то, что инициальный и финальньй отрезки в линейно упорядоченном множестве являются слагаемыми для ординального сложения (см. лемму 6(2) ниже) и что она останется верной даже для произвольного бинарного сложения (одного фиксированного типа) и произвольных реляционных систем. В таком расширенном виде, если выписать отношения между элементами слагаемых, теорема вьглядит так.

ТЕОрема 1. Пусть выполнено условие (*), и для любых $a \in A_{0}, b \in B_{0}, r \in A \backslash A_{0}$ $u s \in B \backslash B_{0}$ выполнено одно из трех следующих условий:

(a) $a \mathscr{R} r \wedge r \mathscr{R} a \wedge b \mathscr{S} s \wedge s \mathscr{S} b$,

(b) $a \mathscr{R} r \wedge \neg r \mathscr{R} a \wedge \neg b \mathscr{S} s \wedge s \mathscr{S} b$,

(c) $\neg a \mathscr{R} r \wedge \neg r \mathscr{R} a \wedge \neg b \mathscr{S} s \wedge \neg s \mathscr{S} b$.

Тогда системы $(A, \mathscr{R})$ и $(B, \mathscr{S})$ изоморфны.

Или, для реляиионных типов, всегда из соотношения $\alpha=\beta+\rho \wedge \beta=\sigma+\alpha$ следует $\alpha=\beta$, где + означает квадратное, ординальное или кардинальное сложсение соответственно случаям (a), (b) $u$ (c).

Примеры. Если $\mathscr{R}$ и $\mathscr{S}$ универсальны и вьполнено условие (a), теорема превращается в теорему Кантора-Бернштейна; то же в случае, когда $\mathscr{R}$ и $\mathscr{S}$ пусты и вьполнено условие $(\mathrm{c})$. Если $\mathscr{R}$ и $\mathscr{S}$ - линейные порядки и вьполнено условие (b), получаем теорему Линденбаума.

Теорема 1 не является новой. Если под $\alpha, \beta, \rho$ и $\sigma$ понимать элементы кардинальной или ординальной алгебры Тарского, то последнее утверждение теоремы вьполняется в этих алгебрах (см. [3, с. 10], [2, с. 17]). Чтобы получить саму теорему, остается заметить, что класс реляционных систем (и класс их типов) образует с операцией кардинального сложения кардинальную алгебру, а с операцией ординального сложения ординальную алгебру (см. [3, с. 248], [2, с. 71, 82]). Для установления последнего факта приходится использовать АС, но на самом деле ничего не теряется, так как доказательство теоремы может быть проведено непосредственно (то же самое верно и для других теорем этих алгебр; см. замечание в [2] на с. 75). Мы помещаем прямое доказательство этой теоремы Линденбаума-Тарского, общее для всех трех случаев. 
Лемма 1. Пусть $f: A_{0} \rightarrow B_{0}, g: A \backslash A_{0} \rightarrow B \backslash B_{0}-$ вложения сужений системы $(A, \mathscr{R})$ в систему $(B, \mathscr{S})$. Тогда отображение $h: A \rightarrow B$, совпадающее $c f$ на $A_{0}$ и с g на $A \backslash A_{0}$, является вложением $(A, \mathscr{R})$ в $(B, \mathscr{S})$, если и только если

$$
\forall x \in A_{0} \quad \forall y \in A \backslash A_{0} \quad(x \mathscr{R} y \Leftrightarrow f(x) \mathscr{S} g(y) \wedge y \mathscr{R} x \Leftrightarrow g(y) \mathscr{S} f(x)) .
$$

\section{ДОКАЗАТЕЛЬСТВО очевидно.}

Пусть $f^{n}$ обозначает $n$-ю итерацию отображения $f$, т.е. $f^{0}$ - тождественное отображение и $f^{n+1}=f \circ f^{n}$ для $0 \leqslant n<\omega$. Через $f A$ обозначим множество $\{f(x): x \in A\}$.

Лемма 2. Пусть $f: B \rightarrow B-$ вложение системы $(B, \mathscr{S})$ в себя $и S \subseteq B \backslash f B$. Положим

$$
P=S \cup f S \cup \cdots \cup f^{n} S \cup \cdots \quad u \quad T=B \backslash P .
$$

Тогда, если

$$
\forall p \in P \quad \forall t \in T \quad(p \mathscr{S} t \Leftrightarrow f(p) \mathscr{S} t \wedge t \mathscr{S} p \Leftrightarrow t \mathscr{S} f(p)),
$$

то $(B, \mathscr{S})$ изоморфна своему сужению на $B \backslash S$.

ДоказАтельство. Согласно лемме 1 отображение $h: B \rightarrow B$, совпадающее с $f$ на $P$ и тождественное на $T$, является вложением $(B, \mathscr{S})$ в себя. Но $S \subseteq B \backslash f B$, так что на самом деле $P=S \sqcup f S \sqcup \cdots \sqcup f^{n} S \sqcup \cdots$ (дизъюнктное объединение); в частности, $f S \sqcup \cdots \sqcup f^{n} S \sqcup \cdots=P \backslash S$. Следовательно, $h P=f S \sqcup \cdots \sqcup f^{n} S \sqcup \cdots=P \backslash S$, и тог да $h B=B \backslash S$. Лемма доказана.

ДоКАЗАТЕЛЬСТВо тЕоРЕМЫ 1. Очевидно, без ограничения общности можно отождествить систему $(A, \mathscr{R})$ с сужением системы $(B, \mathscr{S})$ на $B_{0}$, т.е. считать $A=B_{0}$ и $\mathscr{R}=$ $\mathscr{S} \cap(A \times A)$. Тогда $A_{0}$ отождествится с $f B$ для некоторого вложения $f$ системы $(B, \mathscr{S})$ в себя.

Итак, положим, что $f: B \rightarrow B$ - вложение, $B=S \sqcup f B \sqcup R, A=B \backslash S=f B \sqcup R$, и покажем, что если выполнено одно из условий (a), (b) или $($ c), то система $(B, \mathscr{S})$ изоморфна своему сужению на $B \backslash S$.

Вначале заметим, что для любого $n<\omega$ будет $f^{n} B=f^{n} S \sqcup f^{n+1} B \sqcup f^{n} R$ и $B=S \sqcup f S \sqcup \cdots \sqcup f^{n} S \sqcup f^{n+1} B \sqcup f^{n} R \sqcup \cdots \sqcup f R \sqcup R$.

Положим $P=S \sqcup f S \sqcup \cdots \sqcup f^{n} S \sqcup \cdots$ и $T=B \backslash P$.

Теперь мы покажем, что если выполнено (а), то $B$ является квадратной суммой своих сужений на $P$ и $T$; нужно проверить такую импликацию:

$$
\text { (a) } \Rightarrow \forall p \in P \quad \forall t \in T \quad(p \mathscr{S} t \wedge t \mathscr{S P}) \text {. }
$$

В самом деле, $p \in f^{n} S$ для некоторого $0 \leqslant n<\omega$. Так как $T \subseteq f^{n+1} B \sqcup f^{n} R \sqcup \cdots \sqcup$ $f R \sqcup R$, то достаточно рассмотреть следующие два случая.

Случай 1 . Точка $t$ принадлежит множеству $f^{n-k} R$ для некоторого $1 \leqslant k \leqslant n$.

По условию (a), $\forall a \in f B \forall r \in R(a \mathscr{S} r \wedge r \mathscr{S} a)$. Но при вложении $f^{n-k}$ множество $f B$ переходит в $f^{n-k+1} B$, а его дополнение $R$ до множества $A$ - в $f^{n-k} R$; следовательно, то же верно и для $p \in f^{n} S \subseteq f^{n-k+1} B$ и $t \in f^{n-k} R$.

Случай 2 . Точка $t$ принадлежит множеству $f^{n+1} B \sqcup f^{n} R$.

По условию (а), $\forall b \in f B \sqcup R \forall s \in S(b \mathscr{S} s \wedge s \mathscr{S} b)$. Но при вложении $f^{n}$ множество $S$ переходит в $f^{n} S$, а его дополнение $f B \sqcup R-$ в $f^{n+1} B \sqcup f^{n} R$; следовательно, то же верно и для $p \in f^{n} S$ и $t \in f^{n+1} B \sqcup f^{n} R$. 
Аналогично доказываются две другие импликации:

$$
\begin{aligned}
& \text { (b) } \Rightarrow \forall p \in P \quad \forall t \in T \quad(p \mathscr{S} t \wedge \neg t \mathscr{S} p) \text { и } \\
& \text { (c) } \Rightarrow \forall p \in P \quad \forall t \in T \quad(\neg p \mathscr{S} t \wedge \neg t \mathscr{S} p),
\end{aligned}
$$

дающие аналогичные утверждения для ординальной и кардинальной сумм.

Так как $\forall p(p \in P \Leftrightarrow f(p) \in P)$, получаем, что в любом из случаев (a), (b) или (c) условие леммы 2 вьполнено. Применение этой леммы завершает доказательство.

ЗАмЕчАниЕ. Мы доказали даже нечто большее, именно, аналог теоремы Банаха (см. замечание в конце п. 1 ): если даны вложения $f$ и $g$, отображающие соответственно $A$ на $B_{0}, A_{0}$ на $B$, причем вьполнены условия $(\mathrm{a}),(\mathrm{b})$ или $(\mathrm{c})$, то искомьй изоморфизм $h$ можно составить из них, заставив его на некоторой части совпадать $\mathrm{c} f$, а на ее дополнении - c $g$. Ясно также, что если $f$ и $g$ принадлежат классу, замкнутому относительно суперпозиций и счетного объединения непересекающихся отображений (например, классу всех борелевских отображений), то $h$ тоже будет принадлежать этому классу.

2. Приложение к самоподобным типам. Теперь применим теорему 1 для получения одного интересного факта. В п. 2 мы будем рассматривать только сложение по системе $(B, \mathscr{S})$, где $\mathscr{S}$ - линейньй порядок на $B$, и в частности, (бинарное) ординальное сложение, которое будем обозначать через +. Здесь мы будем, как обычно, вместо $(B, \mathscr{S})$ писать просто $B$ и соответственно вместо $\sum_{(B, \mathscr{S})} \alpha_{b}-$ просто $\sum_{b \in B} \alpha_{b}$. В основном в этом пункте мы говорим о типах. Мы делаем это для краткости; конечно, всюду здесь “типы" можно заменить на “системы".

Тип $\alpha$ назьвается неразложимым (относительно ординального сложения), если всегда из $\alpha=\beta+\gamma$ следует $\beta=0$ или $\gamma=0$ (см. [2, с. 78]).

ПримеРЫ. Среди порядковых типов неразложимы только два: 0 и 1. Кардинальная сумма (двух и более) произвольных типов неразложима; вообще, если тип системы $(B, \mathscr{S})$ неразложим, и $B$ состоит более, чем из одного элемента, то сумма произвольных типов по $(B, \mathscr{S})$ неразложима.

По теореме Йонсона-Тарского, каждый тип а единственным образом разлагается в сумму $\alpha=\sum_{b \in B} \alpha_{b}$ неразложимых типов $\alpha_{i}$ по линейно упорядоченному множеству $B$ (см. [2, с. 78]). Назовем такое разложение каноническим. (Общее определение неразложимости относительно некоторого класса типов и общую теорему Йонсона об единственности разложения см. в [7, с. 100, 109].)

Линейно упорядоченное множество $B$ без крайних точек (если они есть) обозначим через $\langle B\rangle$; и для произвольного типа $\alpha$ с каноническим разложением $\alpha=\sum_{b \in B} \alpha_{b}$ положим $\langle\alpha\rangle=\sum_{b \in\langle B\rangle} \alpha_{b}$.

Назовем тип $\alpha$ cамоподобным, если всегда из того, что $\alpha=\alpha^{\prime}+\beta+\alpha^{\prime \prime}$ и $\beta$ разложимый, следует $\beta=\beta^{\prime}+\langle\alpha\rangle+\beta^{\prime \prime}$ для некоторых $\beta^{\prime}, \beta^{\prime \prime}$. Если, более того, в этой ситуации всегда будет $\langle\beta\rangle=\langle\alpha\rangle$, назовем тип $\alpha$ сильно самоподобным.

НЕкоторЫЕ свойствА И ПРИмеРЫ. Порядковые типы множеств $\mathbb{Q}$ всех рациональных и $\mathbb{R}$ всех действительных чисел сильно самоподобны. Порядковые типы лексикографически упорядоченного множества всех $\omega$-последовательностей счетных ординалов и его всюду плотного подмножества, состоящего из $\omega$-последовательностей, финально равных 0, самоподобны, но не сильно самоподобны, так как в них есть отрезки 
различной конфинальности, следовательно не изоморфные. Любой самоподобньй тип плотен. Пусть $\beta$ (сильно) самоподобен; тогда $\alpha \cdot \beta$ таков же, если и только если $\alpha$ неразложим. Если $\alpha$ с каноническим разложением $\alpha=\sum_{b \in B} \alpha_{b}$ (сильно) самоподобен, то таковы же будут порядковые тип множества $B$ и тип суммы $\alpha=\sum_{b \in I} \alpha_{b}$ по любому отрезку $I$ в $B$. Пополнение (сильно) самоподобного порядкового типа таково же.

Самоподобные типы возникают, в частности, при исследовании умножения типов; порядковые самоподобные типы подробно рассматриваются в [8]. Факт, который мы собираемся доказать, состоит в следующем: для любого самоподобного типа $\alpha=$ $\alpha^{\prime}+\beta+\alpha^{\prime \prime}=\gamma^{\prime}+\delta+\gamma^{\prime \prime}$, типь $\gamma$ и $\delta$ равны, как только их каноничекие разложения являются суммами по линейно упорядоченным множсествам счетной коинициальности и конфинальности. Для порядковых типов это имеет более простую формулировку: все отрезки счетной коинициальности и конфинальности изоморфны (отрезки линейно упорядоченного множества являются его слагаемыми; см. лемму $5(2)$ ниже).

Теорема 2. $\left(\mathrm{AC}_{\omega}\right)$ Пусть $\alpha$ - самоподобный тип, $\alpha=\sum_{b \in B} \alpha_{b}-$ его каноническое разложсние, I и $J$ - отрезки в множестве $B$, имеющие счетную коинициальность и конфинальность. Тогда

$$
\sum_{b \in I} \alpha_{b}=\sum_{b \in J} \alpha_{b}
$$

Лемма 3. Всегда если $\alpha=\gamma+\alpha+\beta+\alpha+\delta$, mo $\alpha=\alpha+\beta+\alpha$.

ДоКАЗАТЕЛЬСТВо прямо следует из теоремы $1(\mathrm{~b})$.

ДоКАЗАТЕЛЬСТВо ТЕОРЕМЫ 2. Без ограничения общности можно считать $\alpha=\langle\alpha\rangle$. Так как из условия видно, что $\alpha$ разложимьй, то используя лемму 3 , положим $\alpha=\alpha+$ $\beta+\alpha$ для какого-нибудь $\beta$. Пусть, как обычно, $\zeta$ обозначает порядковьй тип множества $\mathbb{Z}$ всех целых чисел. Мы покажем, что если отрезок $I$ в $B$ содержит коинициальное и конфинальное подмножество, имеющее тип $\zeta$, то

$$
\sum_{b \in I} \alpha_{b}=(\alpha+\beta) \cdot \zeta
$$

Так как $I$ имеет счетную коинициальность и конфинальность, мы можем взять некоторое подмножество в $I$, коинициальное и конфинальное с ним и изоморфное $\mathbb{Z}$. Тогда мы имеем представление типа $\sum_{b \in I} \alpha_{b}$ в виде некоторой суммы $\zeta$ разложимых слагаемых $\gamma_{j}, j \in \mathbb{Z}$. Заметим, что любое из них самоподобно и разлагается в сумму $\gamma_{j}=\gamma_{j}^{\prime}+\alpha+\beta+\alpha+\gamma_{j}^{\prime \prime}$ для некоторых $\gamma_{j}^{\prime}, \gamma_{j}^{\prime \prime}$. Более того, по $\mathrm{AC}_{\omega}$ мы имеем функцию выбора на счетном семействе всех (непустых) множеств разложений $\gamma_{j}$; т.е. эти разложения могут быть выбраны одновременно. Мы получаем

$$
\sum_{j \in \mathbb{Z}} \gamma_{j}=\sum_{j \in \mathbb{Z}}\left(\gamma_{j}^{\prime}+\alpha+\beta+\alpha+\gamma_{j}^{\prime \prime}\right) .
$$

По лемме 3 для любого $j \in \mathbb{Z}$ будет $\alpha+\gamma_{j}^{\prime \prime}+\gamma_{j+1}^{\prime}+\alpha=\alpha$. Следовательно, $\sum_{b \in I} \alpha_{b}=\sum_{j \in \mathbb{Z}} \gamma_{j}=\sum_{j \in \mathbb{Z}}\left(\gamma_{j}^{\prime}+\alpha+\beta+\alpha+\gamma_{j}^{\prime \prime}\right)=\sum_{j \in \mathbb{Z}}\left(\alpha+\gamma_{j}^{\prime \prime}+\gamma_{j+1}^{\prime}+\alpha+\beta\right)=(\alpha+\beta) \cdot \zeta$.

Теорема доказана. 
ЗАмЕчАниЕ. Можно показать, что таким свойством обладает только счетньй кардинал: для любого несчетного регулярного кардинала $\kappa$ можно построить самоподобный порядковый тип $\alpha$ такой, что $\alpha=\beta+\gamma, \beta \neq \alpha$, и $\alpha$ и $\beta$ имеют одну и ту же конфинальность $\kappa$ (см. [8]; доказательство основано на соображениях стационарности).

Порядковьй тип (иногда) называют коротким, если в него не вкладываются ни $\omega_{1}$, ни $\omega_{1}^{*}$ (см. $[5$, с. 88]).

НЕКОТОРЫЕ СВОЙстВА И ПРИМЕРЫ. Тип множества действительных чисел короткий. Если $\alpha$-счетньй ординал, а тип множества $B$ короткий, то таков же и тип лексикографически упорядоченного множества $\alpha$-последовательностей элементов $B$. Из $\mathrm{AC}_{\omega}$ легко следует, что любое множество несчетного короткого типа содержит подмножество типа $\eta$ множества $\mathbb{Q}$. Теорема Урысона-Хаусдорфа говорит, что (при АС) короткие типы не очень большие: если линейно упорядоченное множество короткого типа содержит вполне упорядочиваемое всюду плотное подмнохество, то его мощность не превосходит $2^{\aleph_{0}}$ (см. [5, с. 166]); оригинальная версия использует АС вместо вполне упорядочиваемости).

СлЕДСТВИЕ. (1) $\left(\mathrm{AC}_{\omega}\right)$ Для того чтобы самоподобный тип а с каноническим разлохсением $\alpha=\sum_{b \in B} \alpha_{b}$ был сильно самоподобен, необходимо и достаточно, чтобы тип мнохсества $B$ был коротким.

(2) (АС) Если в данном классе типов неразложимые типь образуют множество мощности $\kappa \geqslant 2$, то сильно самоподобные типы этого класса такжсе образуют мнохсество, мощность которого не превосходит $\kappa^{2^{\aleph_{0}}}$.

ДокАЗАТЕЛЬСТво. (1) Необходимость очевидна из того, что отрезки разной конфинальности не могут быть изоморфны; достаточность следует из теоремы 2.

(2) Для оценки мощности нужно воспользоваться теоремой Урысона-Хаусдорфа.

ЗАмЕчАнИЕ. В теореме 2 (и в (1) из ее следствия) можно опустить $\mathrm{AC}_{\omega}$, если предположить, что пополнение множества $B$ вполне упорядочиваемо. (В самом деле, тогда вполне упорядочиваемо множество всех разложений слагаемых $\gamma_{j}$. Для каждого $j$ возьмем наименьшее.)

3. Свойство Кантора-Бернштейна. Теперь мы рассмотрим класс релящионных систем, для которых вьполняется непосредственньй аналог теоремы Кантора-Бернштейна, т.е. условие $(*)$ влечет изоморфизм систем. Скажем, что система (и ее тип) обладает свойством Кантора-Бернштейна, если любая ее подсистема, в которую она вкладьвается, изоморфна ей. Понятно, что на классе таких систем предпорядок по вложению является порядком. Мы можем еще обобщить это понятие, введя индекс Кантора-Бернштейна $i(\tau)$ - число типов, эквивалентньх по вложению данному типу $\tau$. Тогда $\tau$ обладает свойством Кантора-Бернштейна, если и только если $i(\tau)=1$.

НЕКОТОРЫЕ СВоЙСТВА И ПРИМЕРЫ. Системы с пустым или универсальным отношением обладают свойством Кантора-Бернштейна. Все ординалы и все типы вида $\alpha+\beta^{*}$ и $\alpha^{*}+\beta$, где $\alpha$ и $\beta$ - ординалы, обладают этим свойством. Некоторые другие разреженные порядковые типы такие, как $\omega+\zeta$ и $\omega^{*} \cdot \zeta$, тоже обладают им. Однако, как легко видеть, $i\left(\omega^{*} \cdot \omega\right)=\aleph_{0}$ и $i(\zeta \cdot \omega)=2^{\aleph_{0}}$. Поэтому даже класс порядковых типов со свойством Кантора-Бернштейна не замкнут относительно (бинарного ординального) умножения. С другой стороны, как показьвает теорема 4 ниже, плотные порядковые типы образуют класс, замкнутьй относительно арифметических операций. 
Так как $\omega^{*} \cdot \zeta=\left(\omega^{2}\right)^{*}+\omega^{*} \cdot \omega$, это свойство не наследуется слагаемыми. Если $\tau$ или $\tau^{*}$ - ординал, то $i(\tau \cdot \zeta)=1$; однако уже $i\left(\zeta^{2}\right)=2^{\aleph_{0}}$. Тип $\tau \cdot \omega$ имеет свойство Кантора-Бернштейна, если и, по $\mathrm{AC}_{\omega}$, только если $\tau$ - ординал. (В самом деле, пусть $\tau-$ не ординал; применяя $\mathrm{AC}_{\omega}$, видим, что в него вкладьвается $\omega^{*}$, и тогда типы $1+\tau \cdot \omega$ и $\omega^{*}+\tau \cdot \omega$ оба эквивалентны типу $\tau \cdot \omega$, но, очевидно, различны.) Следующий простой пример показывает, что (даже счетные) фундированные частичные порядки могут не обладать этим свойством: пусть $X=\{(i, n): i<2, n<\omega\}$ и $(i, m)<(j, n) \Leftrightarrow$ $(i=j=0 \wedge m<n) \vee(i=0 \wedge j=1 \wedge m=n)$; легко видеть, что $i(X)=2^{\aleph_{0}}$. Среди плотных линейных порядков также существуют обладающие свойством Кантора-Бернштейна; но, как показывает лемма 6(2) ниже, они обладают и более сильным свойством точности. Счетно полные булевы алгебры обладают свойством Кантора-Бернштейна (см. [3]).

Рассмотрим еще два близких свойства: точность и жесткость.

Система (и ее тип) точная, если она не изоморфна никакой своей (собственной) подсистеме; иначе говоря, если любое ее вложение в себя является автоморфизмом.

НЕКотоРЫЕ СвоЙствА И ПРИмЕРЫ. Очевидно, каждая точная система обладает свойством Кантора-Бернштейна. Любой бесконечньй ординал показьвает, что обратное неверно. Так как вложения по определению суть инъекции, то все системы, конечные по Дедекинду, точны (множество конечно по Дедекинду, если оно не равномощно никакому собственному подмножеству; см., например, [16, с. 91, 92, 97 и 100]). Обратно, среди систем с пустьм или универсальньм отношением точными будут только конечные по Дедекинду. Т.е. точность может пониматься как своего рода аналог конечности по Дедекинду для произвольных реляционных систем. Простейший пример бесконечной по Дедекинду точной системы доставляет счетная система $(\mathbb{Z}, \mathscr{R})$ с $\mathscr{R}=\{(j, j+1): j \in \mathbb{Z}\}$. (Ее сужение на любое собственное подмножество $A \subset \mathbb{Z}$ имеет такую точку $x \in A$, что $\neg x \mathscr{R} y$ для всех $y \in A$, а вся система не имеет таких точек, следовательно, не изоморфна ему. Легко проверить также, что любая ее бесконечная собственная подсистема не точна.) Душник и Миллер показали, что счетных точных линейно упорядоченных множеств не существует (см. [5, с. 71]); они же построили несчетное (фактически, $2^{\aleph_{0}}$-плотное) точное подмножество действительной прямой (см. [5], [9]-[13]; ср. вопрос 1 ниже). Существуют непрерьвные линейно упорядоченные точные множества (см. [14], [15]); легко видеть, что их всюду плотные подмножества тоже будут точными.

Далее, система (и ее тип) жесткая, если она не имеет нетождественных автоморфизмов. Таким образом, система будет одновременно жесткой и точной, если и только если она не имеет нетождественных вложений в себя.

НЕКотоРЫЕ СВоЙствА И ПРИмЕРЫ. Лемма 6(1) замечает, что все точные линейные порядки являются жесткими; но для произвольных отношений это не верно: простейший контрпример доставляет циклическое отношение на 3 . Точная система $(\mathbb{Z}, \mathscr{R})$ из предыдущего примера также не является жесткой. Любой бесконечньй ординал жесткий и обладает свойством Кантора-Бернштейна, но не точный; то же касается порядковых типов $\alpha+\beta^{*}$, где $\alpha$ и $\beta$-ординалы, хотя бы один из которых бесконечен. Тип $\zeta$ имеет свойство Кантора-Бернштейна, но не жесткий. Тип $\sum_{q \in \mathbb{Q}} f(q)$, где $\mathbb{Q}$ - множество рациональных чисел, $f: \mathbb{Q} \rightarrow \omega$-инъекция, наоборот, жесткий, но не имеет свойства Кантора-Бернштейна. Последний пример показьвает также, что тип, эквивалентньй по вложению жесткому, сам не обязан быть таковьм. Класс жестких порядковых типов не замкнут даже относительно умножения на ординал 2 : тип $\omega+\omega^{*}$ жесткий, но тип 
$\left(\omega+\omega^{*}\right) \cdot 2=\omega+\zeta+\omega^{*}$ не жесткий. Следующий простой критерий предлагает Морель (см. [5, с. 133]): порядковый тип а жсесткий, если и только если его нельзя представить в виде $\alpha=\beta+\gamma \cdot \zeta+\delta$, где + и · означают ординальные сложение и умножение. Очевидно, если $(A, \mathscr{R})$ имеет свойство Кантора-Бернштейна (соответственно точная, жесткая), то $\left(A, \mathscr{R}^{-1}\right)$ имеет то же самое свойство.

Примеры выше показьвают, что эти три свойства независимы и не очень устойчивы относительно “естественных" операций. В этом пункте мы попытаемся больше понять о строении таких систем и их устойчивости. $\mathrm{K}$ сожалению, в этом направлении я имею только частичные результаты. Оставшиеся вопросы помещены в конце этого раздела.

В следующей теореме мы рассматриваем, как связаны точность суммы и произведения с точностью слагаемых и множителей (для линейных порядков это рассмотрено в $[12$, с. 342$]$ и $[11$, с. 559$])$.

ТЕОрема 3. (1) Если сумма $\sum_{(B, \mathscr{S})}\left(A_{b}, \mathscr{R}_{b}\right)$ точная, то и все слагаемые $\left(A_{b}, \mathscr{R}_{b}\right)$ точны.

(2) Eсли $(A, \mathscr{R})$ и $(B, \mathscr{S})$ точные, то и их ординальная сумма $(A, \mathscr{R})+(B, \mathscr{S})$ точна.

(3) Если ординальное произведение $(A, \mathscr{R}) \cdot(B, \mathscr{S})$ точное, то и множсители $(A, \mathscr{R})$ u $(B, \mathscr{S})$ точны.

(4) Если системы $(A, \mathscr{R})$ и $(B, \mathscr{S})$ точные, и кроме того, отношение $\mathscr{S} \cup \Delta$ плотно, связно и антисимметрично, то и их ординальное произведение $(A, \mathscr{R})$. $(B, \mathscr{S})$ точно.

На простых примерах легко убедиться, что точность суммы $\sum_{(B, \mathscr{S})}\left(A_{b}, \mathscr{R}_{b}\right)$ не гарантирует, вообще говоря, точности системы $(B, \mathscr{S})$, по которой слагаемые суммируются. (Однако, если все $\left(A_{b}, \mathscr{R}_{b}\right)$ изоморфны некоторой системе $(A, \mathscr{R})$ и мы можем взять эти изоморфизмы одновременно (например, по АC), такая сумма будет изоморфна ординальному произведению $(A, \mathscr{R}) \cdot(B, \mathscr{S}) ;$ тогда $(B, \mathscr{S})$ будет точной в силу $(3)$.) Очевидно, утверждения (2), (3) и (4) можно по индукции распространить на конечные ординальные суммы и произведения.

Лемма 4. Пусть $(C, \mathscr{T})=(A, \mathscr{R})+(B, \mathscr{S})-$ ординальная сумма $и f: C \rightarrow C-$ вложсение системы $(C, \mathscr{T})$ в себя. Тогда $f A \subseteq A$ или $f B \subseteq B$.

ДоказАтельство. Пусть $f A \nsubseteq A$, т.е. $\exists x \in A(f(x) \in B)$. Так как $x \in A$, из определения ординальной суммы следует, что $\forall b \in B(x \mathscr{T} b \wedge \neg b \mathscr{T} x)$, и так как $f$-вложение, $\forall b \in B(f(x) \mathscr{T} f(b) \wedge \neg f(b) \mathscr{T} f(x))$. Допустим $\exists b \in B(f(b) \in A)$. Тогда в силу условия $f(x) \in B$ опять из определения ординальной суммы следует $f(b) \mathscr{T} f(x) \wedge \neg f(x) \mathscr{T} f(b)$, - противоречие. Следовательно, $\forall b \in B(f(b) \in B)$. Лемма доказана.

Опять по индукции видим, что здесь ординальную сумму двух систем можно заменить на конечную сумму $\sum_{(B, \mathscr{S})}\left(A_{b}, \mathscr{R}_{b}\right)$, если $\mathscr{S}$ - линейньй порядок; однако если $\mathscr{S} \cup \Delta-$ произвольное антисимметричное связное отношение на конечном $B$, лемма может стать неверной: контрпример доставляет циклическое отношение на 3 (ср. вопрос 6 ниже).

Следующая лемма говорит, что в некоторых условиях слагаемые будут отрезками, а отрезки - слагаемыми.

ЛЕмма 5. (1) Если отношение $\mathscr{S} \cup \Delta$ антисимметрично, то все слагаемые $\left(A_{b}, \mathscr{R}_{b}\right)$ в сумме $(C, \mathscr{T})=\sum_{(B, \mathscr{S})}\left(A_{b}, \mathscr{R}_{b}\right)$ являются ее отрезками. 
(2) Если $\mathscr{T} \cup \Delta$ антисимметрично и связно, то любой финальный отрезок $\left(A_{1}, \mathscr{R}_{1}\right)$ в системе $(C, \mathscr{T})$ является слагаемым в ординальной сумме $(C, \mathscr{T})=$ $\left(A_{0}, \mathscr{R}_{0}\right)+\left(A_{1}, \mathscr{R}_{1}\right)$, əде $A_{0}=\left\{x \in C: \forall y \in A_{1}(x(\mathscr{T} \backslash \Delta) y)\right\}$, и отношение $\mathscr{R}_{0}$ есть сужение отношения $\mathscr{T}$ на $A_{0}$.

Если, более того, $\mathscr{T} \cup \Delta-$ линейный порядок, то любой отрезок $\left(A_{1}, \mathscr{R}_{1}\right)$ в системе $(C, \mathscr{T})$ является слагаемым в ординальной сумме $(C, \mathscr{T})=\left(A_{0}, \mathscr{R}_{0}\right)+\left(A_{1}, \mathscr{R}_{1}\right)$ $+\left(A_{2}, \mathscr{R}_{2}\right)$, где $A_{0}$ u $\mathscr{R}_{0}$ определены как раньше, $A_{2}=\left\{x \in C: \forall y \in A_{1}(y(\mathscr{T} \backslash \Delta) x)\right\}$ и $\mathscr{R}_{2}$ - сужение $\mathscr{T}$ на $A_{2}$.

ДокаЗАтельство. (1) Пусть $x, y \in A_{b^{\prime}}, z \in A_{b^{\prime \prime}}$ и $x \mathscr{T} z \wedge z \mathscr{T} y$. Так как $\mathscr{S} \cup \Delta$ антисимметрично, допуская $b^{\prime} \neq b^{\prime \prime}$, получаем $\neg b^{\prime} \mathscr{S} b^{\prime \prime} \vee \neg b^{\prime \prime} \mathscr{S} b^{\prime}$, и тогда по определению суммы $\neg x \mathscr{T} z \vee \neg z \mathscr{T} y,-$ противоречие.

(2) Множества $A_{i}$ не пересекаются в силу антисимметричности отношения $\mathscr{T} \cup \Delta$, и дают в объединении все $C$ в силу его связности. Кроме того, легко проверяется, что отношение между элементами различных $A_{i}$ не меняется от замены их на другие элементы тех же множеств, и значит, $(C, \mathscr{T})=\sum_{(n, \mathscr{S})}\left(A_{i}, \mathscr{R}_{i}\right)$, причем $\mathscr{S} \cup \Delta$ тоже будет антисимметричным и связным; поэтому, если $n=2$ или если $\mathscr{T}$ - линейный порядок, то $\mathscr{S}$ - тоже линейньй порядок. Лемма доказана.

Разумеется, утверждение, двойственное к первой части (2), справедливо для инициальных отрезков (достаточно перейти к $\mathscr{T}^{-1}$ ). Вторая часть (2) не переносится на произвольные антисимметричные связные отношения; простейший контрпример: система $(4, \mathscr{T})$ с отношением $\mathscr{T}=\{(0,1),(0,3),(1,2),(1,3),(2,0),(2,3)\}$ на множестве $4=$ $\{0,1,2,3\}$, и $A_{1}=\{1\}$. (Очевидно, условия вьполнены: $\mathscr{T} \cup \Delta$ антисимметрично и связно, и $\left(A_{1}, \mathscr{R}_{1}\right)$ - отрезок в $(4, \mathscr{T})$.) В этой ситуации будет $A_{0}=\{0\}, A_{2}=\{2,3\}$, но разложение $(4, \mathscr{T})=\sum_{(3, \mathscr{S})}\left(A_{i}, \mathscr{R}_{i}\right)$ невозможно ни для какого отношения $\mathscr{S}$ на множестве 3 .

ДОКАЗАТЕЛЬСТВО ТЕОРЕМЫ 3. (1), (3) очевидны.

(2) Достаточно применить лемму 4.

(4) Для удобства, вместо ординального произведения $(A, \mathscr{R}) \cdot(B, \mathscr{S})$ мы рассмотрим совпадающую с ним сумму $(C, \mathscr{T})=\sum_{(B, \mathscr{S})}\left(A_{b}, \mathscr{R}_{b}\right)$, где $\left(A_{b}, \mathscr{R}_{b}\right)=A \times\{b\}$ для всех $b \in B$ и $\mathscr{R}_{b}$ определены естественным образом. Пусть $f: C \rightarrow C$ - вложение системы $(C, \mathscr{T})$ в себя. Покажем, что оно может быть только автоморфизмом.

Вначале убедимся, что если точки $x_{0}, x_{1} \in C$ принадлежат разным слагаемым, скажем, $A_{b_{0}}, A_{b_{1}}$ соответственно, то их образы при отображении $f$ тоже принадлежат разным слагаемьм.

В самом деле, допустим противное:

$$
\exists b_{2} \in B \quad\left(f\left(x_{0}\right), f\left(x_{1}\right) \in A_{b_{2}}\right) .
$$

Так как $\mathscr{S} \cup \Delta$ связно, а $b_{0} \neq b_{1}$, то $b_{0} \mathscr{S} b_{1} \vee b_{1} \mathscr{S} b_{0}$; будем считать $b_{0} \mathscr{S} b_{1}$ (иначе переименуем эти точки). Так как $\mathscr{S}$ плотно, то $\exists b \in B\left(b_{0}(\mathscr{S} \backslash \Delta) b \wedge b(\mathscr{S} \backslash \Delta) b_{1}\right)$. Проверим, что тогда $f A_{b} \subset A_{b_{2}}$.

Пусть $x \in A_{b}$. Из определения суммы следует, что $x_{0} \mathscr{T} x \wedge x \mathscr{T}_{1}$; а так как $f-$ вложение, то $f\left(x_{0}\right) \mathscr{T} f(x) \wedge f(x) \mathscr{T} f\left(x_{1}\right)$. По лемме 5(1) при антисимметричном $\mathscr{S} \cup \Delta$ слагаемые являются отрезками; значит $f(x) \in A_{b_{2}}$. Имеем $f A_{b} \subseteq f A_{b_{2}}$. Кроме того, множество $A_{b_{2}} \backslash A_{b}$ непусто (оно содержит точки $f\left(x_{0}\right)$ и $f\left(x_{1}\right)$ ). Так как системы 
$\left(A_{b}, \mathscr{R}_{b}\right)$ и $\left(A_{b_{2}}, \mathscr{R}_{b_{2}}\right)$ изоморфны, а $f$ - вложение, получаем, что в $\left(A_{b_{2}}, \mathscr{R}_{b_{2}}\right)$ есть собственная подсистема, изоморфная ей, т.е. что она не точная, - противоречие. Итак, мы показали, что точки $f\left(x_{0}\right)$ и $f\left(x_{1}\right)$ принадлежат различным слагаемьг.

Теперь, используя это свойство, построим вложение $g: B \rightarrow B$ системы $(B, \mathscr{S})$ в себя. Выберем в каждом $A_{b}$ по точке $x_{b}$ и положим, что $g(b)$ равно такому $b^{\prime}$, что $f\left(x_{b}\right) \in A_{b^{\prime}}$; таким образом, $f\left(x_{b}\right) \in A_{g(b)}$.

(Заметим, что мы не используем АС. Из конструкции видно, что существует семейство, состоящее из (очевидных) изоморфизмов системы $(A, \mathscr{R})$ на каждую систему $\left(A_{b}, \mathscr{R}_{b}\right)$; значит, взяв какую-нибудь точку $x \in A$, мы получим множество всех ее образов относительно этих изоморфизмов; это множество искомое. Более того, сейчас мы убедимся, что на самом деле функция $g$ будет одной и той же при любом выборе точек $x_{b}$.)

Проверим, что $g$ - вложение.

То, что $g$ - инъекция, следует из того, что $x_{b}$ выбраны по одной из различных слагаемых, и из только что доказанного свойства: точки из разных слагаемых переходят в разные слагаемые. Остается проверить, что $b_{0} \mathscr{S} b_{1} \Leftrightarrow g\left(b_{0}\right) \mathscr{S} g\left(b_{1}\right)$. Имеем

$$
b_{0} \mathscr{S} b_{1} \Leftrightarrow x_{b_{0}} \mathscr{T} x_{b_{1}} \Leftrightarrow f\left(x_{b_{0}}\right) \mathscr{T}\left(x_{b_{1}}\right) \Leftrightarrow g\left(b_{0}\right) \mathscr{T} g\left(b_{1}\right)
$$

В самом деле, первая равносильность следует из того, что $x_{b_{0}}$ и $x_{b_{1}}$ принадлежат разным слагаемым и из определения суммы; вторая - из того, что $f$ - вложение; далее, $f\left(x_{b_{0}}\right)$ и $f\left(x_{b_{1}}\right)$ принадлежат соответственно $A_{g\left(b_{0}\right)}$ и $A_{g\left(b_{1}\right)}$ (по построению $g$ ), причем $g\left(b_{0}\right) \neq g\left(b_{1}\right)$ (опять по доказанному вначале свойству), и тогда третья равносильность следует из определения суммы. Итак, мы проверили, что $g$ - вложение системы $(B, \mathscr{S})$ в себя.

Далее, $g$ - ее автоморфизм. Это следует из того, что система $(B, \mathscr{S})$ точная, a $g$ - ее вложение в себя.

Теперь убедимся, что если точки $x^{\prime}, x^{\prime \prime} \in C$ принадлежат одному и тому же слагаемому, скажем, $A_{b}$, то их образы при отображении $f$ тоже принадлежат одному и тому же слагаемому.

В самом деле, допустим противное: $\exists b^{\prime} \neq b^{\prime \prime}\left(f\left(x^{\prime}\right) \in A_{b^{\prime}} \wedge f\left(x^{\prime \prime}\right) \in A_{b^{\prime \prime}}\right)$. Тогда, так как $g$ - перестановка $B$, то $\exists b_{0} \neq b_{1} \quad\left(g\left(b_{0}\right)=b^{\prime} \wedge g\left(b_{1}\right)=b^{\prime \prime}\right)$. По крайней мере одно из $b_{0}, b_{1}$ не равно $b$; пусть, например, $b_{0} \neq b$. Получаем, что $f\left(x_{b_{0}}\right), f\left(x^{\prime}\right) \in A_{b^{\prime}}-$ образы точек из различных слагаемых $A_{b_{0}}, A_{b}$ принадлежат одному и тому же слагаемому $A_{b^{\prime}},-$ противоречие. Итак, мы проверили, что точки из одного слагаемого при отображении $f$ переходят в одно и то же слагаемое.

Значит, $\forall b \in B\left(f A_{b} \subseteq A_{g(b)}\right)$. А так как сужение отображения $f$ на $A_{b}$ является вложением, а все $A_{b}$ изоморфны и точны, то на самом деле

$$
\forall b \in B \quad\left(f A_{b}=A_{g(b)}\right)
$$

причем это сужение будет изоморфизмом из $\left(A_{b}, \mathscr{R}_{b}\right)$ на $\left(A_{g(b)}, \mathscr{R}_{g(b)}\right)$. Прибавляя то, что $g$ - автоморфизм $(B, \mathscr{S})$, окончательно получаем: $f$ - автоморфизм $(C, \mathscr{T})$. Теорема доказана.

Очевидно, (1) и (3) остаются верньми и для жестких систем; на примерах выше мы видели, что (2) и (4) становятся ложными. 
СЛЕДСТВИЕ. Пусть система $(A, \mathscr{R})$ точная. Тогда

(1) если отношение $\mathscr{R} \cup \Delta$ антисимметрично и связно, то все ее инициальные и финальные отрезки точны;

(2) если $\mathscr{R} \cup \Delta$ - линейный порядок, то все ее отрезки точны, а все ее разрежсенные отрезки конечны по Дедекинду. С другой стороны, существуют бесконечные по Дедекинду точные линейно упорядоченные множества без плотных отрезков.

ДокаЗАТЕЛЬСтво. Точность отрезков следует из леммы 5(2) и теоремы 3(1); конечность по Дедекинду разреженных отрезков - из того, что любое счетное (и значит, любое бесконечное по Дедекинду) разреженное линейно упорядоченное множество содержит отрезок либо типа $\omega$, либо типа $\omega^{*}$ (см. [5]). Чтобы построить бесконечное по Дедекинду точное линейно упорядоченное множество без плотных отрезков, достаточно в силу теоремы $3(4)$ взять множество типа $2 \cdot \tau$ при любом бесконечном по Дедекинду плотном и точном $\tau$.

ЛЕмма 6. (1) Если линейно упорядоченное мнохсество точное, то оно жесткое.

(2) Если плотное линейно упорядоченное множество обладает свойством Кантора-Бернштейна, то оно точное (и жесткое).

ДокАЗАТЕЛЬСТво. Пусть множество $A$ (строго) линейно упорядочено отношением $<$ и $f$-его вложение в себя. Предположим, $f$ не тождественно, т.е. $\exists x_{0} \in A\left(x_{0} \neq f\left(x_{0}\right)\right)$. Пусть $A_{1}$ - отрезок, состоящий из всех точек между $x_{0}$ и $f\left(x_{0}\right)$ :

$$
A_{1}=\left\{x \in A: x_{0}<x<f\left(x_{0}\right) \vee f\left(x_{0}\right)<x<x_{0}\right\},
$$

и пусть $A_{0}, A_{2}$ - множества из леммы $5(2)$; согласно этой лемме, $A$ есть ординальная сумма $A=A_{0}+A_{1}+A_{2}$ (я опускаю обозначения отношений). В силу транзитивности $<$, отображение, тождественное на $A_{0}$ и совпадающее с $f$ на $A_{1}+A_{2}$, будет вложением множества $A$ в его собственное подмножество $A_{0}+A_{2}$, что доказьвает (1). Для доказательства (2) предположим, что $A$ плотно; тогда в $A_{1}$ можно взять двухэлементное подмножество, скажем $A^{\prime}$. Тогда $A$ вкладьвается в $A_{0}+A^{\prime}+A_{2}$, но не изоморфно ему, так как последнее не плотно. Лемма доказана.

ТЕОремА 4. Класс плотных линейно упорядоченных множеств, обладающих свойством Кантора-Бернштейна (как и класс их типов) замкнут относительно ординальных сложения, умножсения и обратных к ним операций (там, где последние определены).

ДоКАЗАТЕЛЬСТВо прямо следует из леммы 6(2) и теоремы 3.

4. Вопросы. В этом пункте мы приведем несколькооткрытых вопросов, относящихся к предыдущему пункту. Первая группа вопросов относится к наименьшей возможной мощности бесконечных (по Дедекинду) точных систем.

1. Существует ли счетное точное частично упорядоченное мнохсество? Существует ли реляиионная система со связным отношением? Какова наименьшая мощность бесконечного точного линейно упорядоченного множества?

В последнем вопросе "наименьшая" подразумевает, конечно, АС; эта мощность расположена между $\aleph_{1}$ и $2^{\aleph_{0}}$ и нетривиальньй ответ предполагает ее оценку с помощью 
малых кардиналов. В [13] мы покажем, в частности, что она не превосходит non $(\mathscr{M})$ (наименьшей мощности множества действительных чисел второй категории).

Следуюшие две группы вопросов относятся к сохранению точности и свойства Кантора-Бернштейна.

2. Будут ли точными следующие системы:

кардинальная (квадратная) суммма двух точных систем;

конечная сумма точных систем;

сумма конечных систем по точной системе;

сумма точных систем по точной системе?

3. Будут ли обладать свойством Кантора-Бернштейна следующие системы:

ординальная (кардинальная, квадратная) сумма двух систем с этим свойством;

конечная сумма таких систем;

сумма конечных систем по системе со свойством Кантора-Бернштейна?

Интересно решить вопросы из 2 и 3 хотя бы для линейных порядков. Более слабые версии этих вопросов получатся, если считать все слагаемые одинаковыми, т.е. заменить суммы на ординальные произведения двух систем. Интересно рассмотреть также другие операции над системами, например, кардинальные произведения или суперпозиции.

4. Охарактеризовать разрежсенные линейно упорядоченные мнохества со свойством Кантора-Бернштейна. Верно ли, что необходимым является условие: множество не содержит бесконечного числа неограниченных (т.е. не имеющих крайних точек) отрезков? Или дажее: если $\zeta \cdot \omega$ вкладывается в $\tau$, то $i(\tau) \geqslant 2^{\aleph_{0}}$ ?

Тип $\omega^{*} \cdot \omega$ показывает, что это условие не достаточно.

5. Верно ли, что если $C=A \cup B-$ разреженное линейно упорядоченное множество, а $A$ и $B$ обладают свойством Кантора-Бернштейна, то и С тоже обладает этим свойством?

Для плотных множеств это неверно: действительную прямую можно разбить на два точных подмножества (см. [5, с. 151]).

В следующем вопросе рассматривается возможность дать аналог леммы 4 для произвольных антисимметричных связных отношений на конечном множестве.

6. Пусть $(C, \mathscr{T})=\sum_{(n, \mathscr{S})}\left(A_{b}, \mathscr{R}_{b}\right)$, где $n<\omega$, а отношение $\mathscr{S} \cup \Delta$ антисимметрично и связно, и пусть $f: C \rightarrow C$ - вложение $(C, \mathscr{T})$ в себя. Верно ли, что $\exists b^{\prime}, b^{\prime \prime}<n \quad\left(f A_{b^{\prime}} \subseteq A_{b^{\prime \prime}}\right)$ ?

7. Переносятся ли утверждения леммы 6 на частично упорядоченные множества?

5. $\kappa$-арные отношения. Множество $A \times A$ естественно отождествить с $A^{2}$ (множеством всех отображений из 2 в $A$ ). Это подсказывает назвать $\mathscr{R} \subseteq A^{\kappa} \kappa$-арным отношением (см. [7]). Большая часть понятий легко переносится. Так, диагональ в $A^{\kappa}-$ это множество $\left\{x \in A^{\kappa}: \exists a \in A \forall \alpha<\kappa(x(\alpha)=a)\right\} ;$ вложсение $\kappa$-арной системы $(A, \mathscr{R})$ в $\kappa$-арную систему $(B, \mathscr{S})$ - это такая инъекция $f: A \rightarrow B$, что

$$
\forall x \in A^{\kappa} \quad(x \in \mathscr{R} \Leftrightarrow f \circ x \in \mathscr{S})
$$


сумма $к$-арных систем $\left(A_{b}, \mathscr{R}_{b}\right)$ по $\kappa$-арной системе $(B, \mathscr{S})$, где $A_{b}$ при различных $b \in B$ не пересекаются, - это система

$$
(C, \mathscr{T})=\sum_{(B, \mathscr{S})}\left(A_{b}, \mathscr{R}_{b}\right), \quad \text { где } C=\bigsqcup_{b \in B} A_{b} \quad \text { и } \quad \mathscr{T}=\bigsqcup_{b \in B} \mathscr{R}_{b} \sqcup \bigsqcup_{s \in \mathscr{S} \backslash \Delta} \prod_{\alpha<\kappa} A_{s(\alpha)} \text {, }
$$

и т.п. В этой работе мы не будем подробно рассматривать такие системы и ограничимся одним замечанием, показывающим, что некоторые "хорошие" свойства при переходе к $\kappa>2$ могут теряться. Тривиальная проверка показьвает, что сложение $\kappa$-арных систем по $\kappa$-арной системе остается “обобщенно ассоциативным” при любом $\kappa$ :

$$
\sum_{(C, \mathscr{T})} \sum_{\left(B_{c}, \mathscr{S}_{c}\right)}\left(A_{b_{c}}, \mathscr{R}_{b_{c}}\right)=\sum_{\sum_{(C, \mathscr{T})}\left(B_{c}, \mathscr{S}_{c}\right)}\left(A_{b_{c}}, \mathscr{R}_{b_{c}}\right)
$$

Однако, имеет место

ПРЕДЛОЖЕНИЕ. Бинарное сложение (фиксированного типа) к-арных систем, и даже их типов, неассочиативно при $\kappa>2$, т.е. существуют к-арное отношение $\mathscr{S}$ на множестве 2 и к-арные системы $(A, \mathscr{A}),(B, \mathscr{B}),(C, \mathscr{C})$, для которых $((A, \mathscr{A})+(B, \mathscr{B}))+(C, \mathscr{C})$ не изоморфно $(A, \mathscr{A})+((B, \mathscr{B})+(C, \mathscr{C}))$, где $\left(A_{0}, \mathscr{R}_{0}\right)+\left(A_{1}, \mathscr{R}_{1}\right)$ означает $\sum_{(2, \mathscr{S})}\left(A_{i}, \mathscr{R}_{i}\right)$.

ДокАЗАтЕЛЬСтво. Достаточно построить пример при $\kappa=3$. Пусть $A, B, C$ - (попарно различные) синглетоны, например, $A=\{0\}, B=\{1\}, C=\{2\}$ с пустыми тернарными отношениями $\mathscr{A}, \mathscr{B}, \mathscr{C}$ на них. В качестве $\mathscr{S} \subseteq\{0,1\}^{3}$ возьмем $\{(0,0,1)\}$. Обозначим первую сумму из теоремы через $(D, \mathscr{D})$, а вторую через $(E, \mathscr{E})$. Получаем $D=E=3$,

$$
\mathscr{D}=\{(0,0,1),(0,0,2),(0,1,2),(1,0,2),(1,1,2)\}, \quad \text { и } \quad \mathscr{E}=\{(0,0,1),(0,0,2),(1,1,2)\} .
$$

Предложение доказано.

Заметим, что произведение двух типов $\kappa$-арных систем, определенное аналогично ординальному произведению двух типов бинарных систем, будет ассоциативным в силу "обобщенной ассоциативности" (обобщенного) сложения.

Для $\kappa$-арных отношений аналог теоремы 1 будет верен в случаях кардинального и квадратного $\kappa$-арных сложений (т.е. по системе с пустым и с универсальным $\kappa$-арньп отношением). Было бы интересно рассмотреть другие случаи.

Автор признателен проф. Харцхейму за вопрос о теореме Линденбаума, стимулировавший написание части этой заметки.

\section{СПИСОК ЦИТИРОВАННОЙ ЛИТЕРАТУРЫ}

[1] Куратовский К., Мостовский А. Теория множеств. М.: Мир, 1970.

[2] Tarski A. Ordinal Algebras. Amsterdam, 1956.

[3] Tarski A. Cardinal Algebras. NY: Oxford University Press, 1949.

[4] Dedekind R. Gesammelte mathematische Werke. Braunschweig, 1932.

[5] Rosenstein J. G. Linear orderings. NY, 1982. 
[6] Sierpiński W. Cardinal and Ordinal Numbers. Warsaw, 1958.

[7] Jónsson B. Appendix B // Tarski A. Ordinal Algebras. Amsterdam, 1956.

[8] Савельев Д. И. Самоподобные линейно упорядоченные множества (в печати).

[9] Ginsburg S. Some remarks on order types and decompositions of sets // Trans. Amer. Math. Soc. 1953. V. 74. № 3. P. 514-535.

[10] Ginsburg S. Further results on order types and decompositions of sets // Trans. Amer. Math. Soc. 1954. V. 77. № 1. P. 122-150.

[11] Ginsburg S. Fixed points of products and ordered sums of simply ordered sets // Proc. Amer. Math. Soc. 1954. V. 5. № 4. P. 554-565.

[12] Ginsburg S. Order types and similarity transformations // Trans. Amer. Math. Soc. 1955. V. 79. № 2. Р. 341-361.

[13] Савельев Д. И. О порядковых типах множеств действительных чисел (в печати).

[14] Gillman L. A continuous exact set // Proc. Amer. Math. Soc. 1958. V. 9. № 3. P. 412-418.

[15] Sherrie N. Continuous and exact sets of specified cardinality // Zeit. Math. Log. Grundlag. Math. 1989. V. 35. P. 211-224.

[16] Lévy A. Basic Set Theory. Jerusalem, 1978.

[17] Lindenbaum A., Tarski A. Communication sur les recherches de la théorie des ensembles // C. R. Sci. Varsovie, III. 1926. V. 19. P. 299-330.

ВИнити

E-mail: saveliev@mccme.ru
Поступило

07.03.2000

Исправленный вариант

26.08.2003 